\title{
RF ENERJİ HASATLAMA SİSTEMLERİ İÇİN ÇİFT BANDLI GREİNACHER DOĞRULTUCU DEVRE TASARIMI
}

\author{
Mehmet Ali BELEN*
}

Artvin Çoruh Üniverstiesi, Mühendislik Fakültesi, Elektrik Elektronik Mühendisliği Bölümü, Artvin, Türkiye

\begin{tabular}{l} 
Anahtar Kelimeler \\
\hline RF Enerji Hasatlama, \\
Kablosuz Enerji Transferi, \\
Greinacher Doğrultucu, \\
Rectenna, \\
HSMS2850,
\end{tabular}

\begin{abstract}
Özet
Enerji hasatlama sistemleri günümüzde yeni bir enerji kaynağı olarak adlandırılmakta ve ortamda bulunan mevcut elektromanyetik enerjinin kullanılmasını he deflemektedir. Enerji hasatlama sistemleri, düşük güç tüketimine sahip cihazlar için etkili bir çözüm olarak ortaya çıkmaktadır. Bu sistemlerinin temel amacl, düşük güç tüketimine sahip haberleşme sistemleri (ZigBee teknolojileri ve Kablosuz sensör ağları) ile birlikte kullanılıp batarya ömrünü uzatmaktır. Bu sistemlerin temel bileşenleri şunlardır: (1) minyatür anten ve (2) yüksek verimli doğrultucu devresi. Birinci bileşen ortamdaki RF dalgalarını toplarken, ikincisi ise toplanan RF enerjisini doğrultarak DC'ye çevirir. Bu çalışmada $2.4 \mathrm{GHz}$ ve $2.7 \mathrm{GHz}$ frekanslarında çalışan çift bandlı Greinacher doğrultucu devre tasarımı önerilmiştir. Uyumlandırma katında L-C ve mikroşerit stub yapıları kullanılmıștır. Greinacher doğrultucu devresinin simetrik yapısı, diyotlar tarafından üretilen harmoniğin azalmasına yardımcı olmaktadır. Yapılan simülasyon sonuçlarına göre, $10 \mathrm{dBm}$ giriş gücüne karşılık çıkışta 100 Kohm'luk yük direncinde 4,02V'luk bir DC gerilim elde edilmiştir. 0dBm'lik giriş gücünde ise LTE frekansında $100 \mathrm{ohm}$ ile $100 \mathrm{Kohm}$ arasında değişen yük dirençlerinde -5 ile $-15 \mathrm{dBm}$ arasında güç değerleri elde edilmiştir. Tasarımlar ve spesifikasyonları AWR yazılımında yapılan benzetimler ile elde edilmiştir. Doğrultucudan elde edilen DC gerilim kullanılarak düşük güç tüketimine sahip sensörler ve sensör ağları beslenebilir ya da DC gerilim depolanarak pil ömrü uzatılabilir.
\end{abstract}

\section{DUAL BAND GREINACHER RECTIFIER CIRCUIT DESIGN FOR ENERGY HARVESTING SYSTEMS}

\begin{tabular}{l} 
Keywords \\
\hline RF Energy Harversting, \\
Wireless Energy Transfer, \\
Greinacher Rectifier, \\
Rectenna, \\
HSMS2850,
\end{tabular}

Keywords

Wireless Energy Transfer, Rectenna,

HSMS2850,

\begin{abstract}
Nowadays, the RF energy harvester systems can be named as one of the novel energy source which aims to use the electromagnetic energy in environment. Harvester systems are seen as an effective solution method for low power consumption circuits. These systems can be used with low power consumption communication system (ZigBee and wireless sensors) to extend the battery life. These systems are consist of: (a) a miniaturized antenna, (b) a high performance rectifier circuit. The first component gathers the RF signals in environment while the second component rectifies the signals to a DC current. Herein, design of a dual band Greinacher rectifier circuit for 2.4 and $2.7 \mathrm{GHz}$ applications is studied. L-C type circuits and microstrip stub structures are used for matching layer. The symmetrical structure of Greincacher rectifier provides reduction in harmonics of diodes. As it can be seen from simulation results, for an input power of $10 \mathrm{dBm}$ a voltage of $4.02 \mathrm{~V}$ DC voltage on a $100 \mathrm{Kohm}$ load resistor, and for an input power of $0 \mathrm{dBm}$ with a variable load resistor of $100-100 \mathrm{~K}$ ohm $-5 \sim 15 \mathrm{dBm}$ is obtained. Designs and specifications were obtained by simulations performed in AWRsoftware. Thus, by
\end{abstract}

\footnotetext{
* ilgili yazar / Corresponding author: mehmetalibelen@artvin.edu.tr +90-466-215-4650
} 
using the DC voltage that is rectified by the RF harvester it is possible to supply low power sensors or extend the battery life of the systems.

Alıntı / Cite

Belen M. A. (2018) RF Enerji Hasatlama Sistemleri İçin Çift Bandlı Greinacher Doğrultucu Devre Tasarımı, Journal of Engineering Sciences and Design, 6(2), 348-353.

\section{Yazar Kimliği / Author ID (ORCID Number)}

M. A. Belen, 0000-0001-5588-9407

\begin{tabular}{l|l}
\hline Başvuru Tarihi /Submission Date & 17.02 .2018 \\
Revizyon Tarihi / Revision Date & 05.06 .2018 \\
Kabul Tarihi / Accepted Date & 18.06 .2018 \\
Yayım Tarihi / Published Date & 23.06 .2018 \\
\hline
\end{tabular}

\section{Giriş}

Radyo frekansı (RF) enerjisi, cep telefonları, el telsizleri, mobil baz istasyonları ve televizyon / radyo yayın istasyonları da dahil olmak üzere, milyarlarca radyo vericisinden yayınlanmaktadır (Paradiso, Starner, 2005). RF enerjisini ortamdan veya tahsis edilmiş kaynaklardan toplanması, düşük güçlü cihazların sürekli şarj edilmesini sağlayacak ve batarya ihtiyacını ortadan kaldırabilecek (Gilbert, Balouchi, 2008; Curty, Joehl, 2000). Ayrica, şarj edilebilir ve atılabilir pillerin kullanım ömrünü uzatmak için batarya tabanlı cihazlar şarj edilebilir. Pilsiz cihazlar, isteğe bağlı olarak veya yeterli şarj biriktiğinde çalışmak üzere tasarlanabilir. Her iki durumda da, cihazlar kablosuz enerji ile beslenerek konnektörlerden, kablolardan ve pil içeren panellerden arındırılır. Kablosuz güç iletiminde çevreci RF enerjisinin toplanması konusundaki yoğunlaşma, mobil baz istasyonları ve el cihazları gibi radyo vericilerinin ürettiği 'serbest' enerjinin kullanılmasıdır. RF vericileri, mobil aboneliklerin sayısı arttıkça artmaya devam edecektir (Rebello, 2010). Özellikle 1 milyar'a ulaşan geniş bant mobil aboneliklerinin artması ilgi çekicidir (Cheng, Chawathe, 2005). Ayrıca, dizüstü bilgisayarlar gibi kablosuz cihazların ve WiFi yönlendiricilerin sayısının da gün geçtikçe arttığını göz önünde bulundurulmalıdır. Zaten kentsel bazı ortamlarda tek bir konumdan onlarca WiFi vericisi alglayabilir (Cheng, Chawathe, 2005), kapalı alanda, kablosuz yerel ağ (WLAN veya basitçe WiFi) erişim noktalarında olduğu gibi, $100 \mathrm{~mW}$ 'lk bir güç seviyesinde iletim yapan tipik bir WiFi yönlendiriciden enerji toplayabilirsiniz. Elbette, uzun mesafeli hasat için, mobil baz istasyonlarından ve yayın radyo kulelerinden RF enerjisinin pratik olarak hasat edilmesi için daha yüksek kazanımlara ihtiyaç vardır. Çünkü cep telefonları, RF enerjisini toplayacak geniş bir verici kaynağını temsil etmektedir ve kullanıcı sayısı gün geçtikçe artmaktadır.Ortamdaki RF enerjisi, çok çeşitli düşük güçlü cihazları şarj etmek veya çalıştırmak için kullanılabilir. Yakın mesafeden ( $R F$ vericisine) bu enerji, küresel konumlandırma sistemi veya radio frekansı tanımlama (RFID) izleme etiketleri, giyilebilir medikal sensörler ve elektronik kitap okuyucuları ve kulaklıklar gibi tüketici elektronikleri dâhil bir dizi cihazı şarj etmek için kullanılabilir. Cep telefonları, birtakım pilsiz kablosuz cihazlar için taşınabilir RF güç kaynakları olarak da kullanılabilir. Yani, cep telefonunu kullanırken, vücuda takılan sensörler, WiFi, Bluetooth veya ZigBee gibi yaygın olarak kullanılan protokoller aracılığıyla veri aktarımını etkinleștirmek üzere güçlendirilebilir. Aktarılan veriler daha sonra telefonda yerel olarak görüntülenebilir veya bir hastane veya bakım noktası servis sağlayıcısındaki uzaktan izleme servisine iletilebilir.

Ortamdaki RF enerjisini toplamak için araştırma çabaları 1990'lı yılların sonlarından beri hız kazandı. $\mathrm{Bu}$ ivmenin başlıca nedeni RF verici cihazların büyümesi ve düşük güç tüketen elektroniğinin yaygınlaşmasıdır. Hagerty (Hagerty, Helmbrecht, 2006), önceki çalışmaları arasında çevre RF gücünü 2$18 \mathrm{GHz}$ frekans aralığında hasat etmeye çalışan geniş bantlı bir anten dizisi sunmuştu. Ayrıca, 2009 yılında Intel Research Seattle, 960 kW'lık bir TV yayın istasyonunu kullanarak $4.1 \mathrm{~km}$ uzaklığa RF enerjisinin hasat edildiğini gösterdi (Sample, A., Smith, 2009). Powercast, 2005 yllında benzer bir çalışma gerçekleştirerek daha küçük bir güç (5 kW AM) radyo istasyonu (Ostaffe, 2010) kullanarak 2,4 km uzaklıktan RF enerjiyi başarılı bir şekilde hasatlamıștır. Bununla birlikte, bu sistemlerde yapılan ölçümler açık alanda alıcı ver vericinin görüş alanları açıkken yapılmış hasatlayıcı devrenin boyutu fiziksel olarak büyük, yüksek kazançlı anten kullanılarak gerçekleştirilmiştir. Görüş alanının kısıtlanması RF sinyal seviyesinde düşmeye sebep olacaktır. Anten boyutunun minimize edilmeside kazancı düşüreceğinden yine elde edilebilecek gücün düşmesine sebep olacaktır.

Bu çalışmada ISM ve LTE bandlarının yoğun olarak kullanıldığı ortamlardaki düşük güç tüketimine sahip cihazlara yönelik $2.4 \mathrm{GHz}$ ve $2.7 \mathrm{GHz}$ frekanslarında çalışan çift bandlı greinacher doğrultucu devre tasarımı önerilmiştir. Böylece düşük güç tüketimine sahip haberleşme sistemleri için batarya sorununu çözecek bir yöntemdir. Tüketici elektroniği (mobil cihazlar) ve endüstriyel alanda (kablosuz sensör şebekeler, tıbbi ve askeri cihazlar) bir çok uygulamada kullanilabilecektir. 


\section{RF Enerji Hasatlayıcı Devre Tasarımı}

$\mathrm{RF}$ enerji toplama sistemi, anten, empedans uyumlandırma, RF doğrultucu devresi, DC filtre devresi ve yükten oluşur. RF enerjisi anten tarafından alınır ve daha sonra alıcı sinyal alıcı anten ile RF sinyalini DC çıkış gücü haline dönüştüren RF doğrultucu devresi arasında eşleşecek şekilde kaynakçekme tekniği ile tasarlanan empedans eşleştirmesi yoluyla gönderilir. DC çıkış gücü, Şekil 1'de gösterildiği gibi yüke göndermeden önce DC filtre devresi tarafından filtrelenir. Bu çalışmada, RF doğrultucu, empedans uyumlandırma ve 50 ohm girişi destekleyecek şekilde tasarlanmıștır.

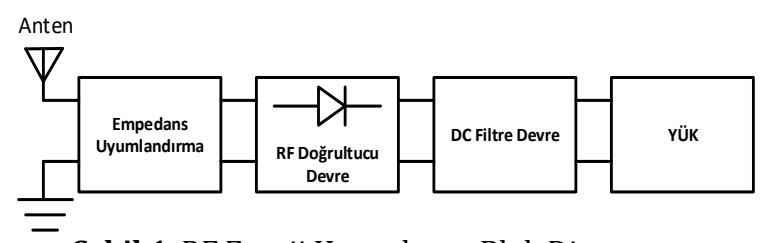

Şekil 1. RF Enerji Hassatlayıcı Blok Diagramı

\subsection{Uyumlandırma Katı Tasarımı}

Enerji toplama devresinin en önemli şartlarından biri ortamdan alınan alınan gücü anten tarafından doğrultucu devreye aktarmaktır. Bu kısımda anten ile doğrultucu devre kısmında empedans uyumsuzlukları meydana gelebilir bunun en önemli nedeni diyotun doğrusal olmayan çalışma karakteristiğidir. $\mathrm{Bu}$ durumda, devrenin performansı doğrultucu ve anten arasında uyumlandırma katının devreye sokulmasıyla kontrol edilebilir. RF sinyal girişinde uyumlandırma sağlanmadığında, bir miktar enerji yansır, bu yansıyan güç, çıkış voltajında azalmaya yol açar, kaynak ve yük arasındaki iletim hattında duran dalgalar oluşturur. Uygun uyumlandirma, uygun uyumlandırma topolojisi ve bileșen değerleri seçimi ile mümkündür. Uyumlandırma katındaki ufak bir değişiklik, enerji dönüşümünün etkinliğinin maksimum olduğu frekans aralığını büyük ölçüde değiştirir. Bu çalışma kapsamında L-C ve mikroşerit stublar kullanılarak uyumlandırma sağlanmıștır.

\subsection{Schottky Diyot Seçimi}

$\mathrm{RF}$ enerjileri genellikle düşük güç alanlarından olduğundan, bu bölgedeki sinyallerin tepe voltajı doğrultucu diyotun açılma geriliminden (H. Yan, J. G. M. Montero, 2005) çok daha küçüktür. Bu gereksinimi karşılamak için çok düşük voltaj gerilimi ve yüksek anahtarlama hızı gereklidir. Düşük gerilim düşüşünden dolayı, ısı olarak daha az enerji harcanır ve verimlilik açısından hassas uygulamalar için en verimli seçimdir. Schottky diyot düşük ileri gerilimi ve yüksek anahtarlama hızı sunar ve RF enerji toplama için ideal bir bileşen olarak düşünülür. RFID uygulamalarında schottky diyot doğrultucu devreleri yaygın olarak kullanılmaktadır. Schottky diyot girişine uygulanan $\mathrm{RF}$ dalgalar doğrultularak $\mathrm{DC}$ 'ye doğrultulmaktadır. RF enerji hasatlama uygulamaları için kullanılacak schottky diyotlar düşük barrierli olmalıdır. Doğru diyot seçimi ile yüksek hasssasiyetli, yüksek verimli ve az kayıplı doğrultma gerçekleşmektedir. Bu çalışmada $2.4 \mathrm{GHz}$ ve $2.7 \mathrm{GHz}$ frekanslarında çalışacak toplu (lumped) elemanlar ile HSMS2850 (düşük yüzey kayıplı) schottky diyot modeli kullanılarak greinacher doğrultucu devre tasarımı önerilmiştir.

\subsection{Yük Empedansı Seçimi}

Her elektronik cihazın belirli bir aralıktaki yük empedansı üzerinde çalışması için standart bir şartı vardır, bu belirli aralığın altında ve üstünde cihaz performansı düşecektir. Enerji toplama devreleri, kademelerin sayısına, doğrusal olmayan cihazın tipine ve reaktif bileşenin seçimine göre değişen belirli bir yük empedansı aralığına sahiptir. Bu nedenle, yük empedansı aralığının seçimini ve devre performansı üzerindeki etkisini doğrulamak önemlidir. $\mathrm{Bu}$ çalışmada doğrultucu devresini, hattın yük empedansı değişimine göre performası incelenmiştir.

\subsection{Doğrultucu Tasarımı}

Kablosuz yerel ağ (WLAN veya basitçe WiFi) erişim noktalarında, $100 \mathrm{~mW}$ 'lık bir güç seviyesinde iletim yapan tipik bir $\mathrm{WiFi}$ yönlendiriciden enerji toplayabilirsiniz. Elbette, uzun mesafeli hasat için, mobil baz istasyonlarından ve yayın radyo kulelerinden RF enerjisinin pratik olarak hasat edilmesi için daha yüksek güç kazançlarına ihtiyaç vardır. Bu amaca yönelik olarak, tek kademeli tam dalga greinacher doğrultucunun (Curty, Joehl, 2000; Olgun, Chen, 2012' den) modifiye edilmiş bir versiyonunu şekil 2'de gösterilmiştir.

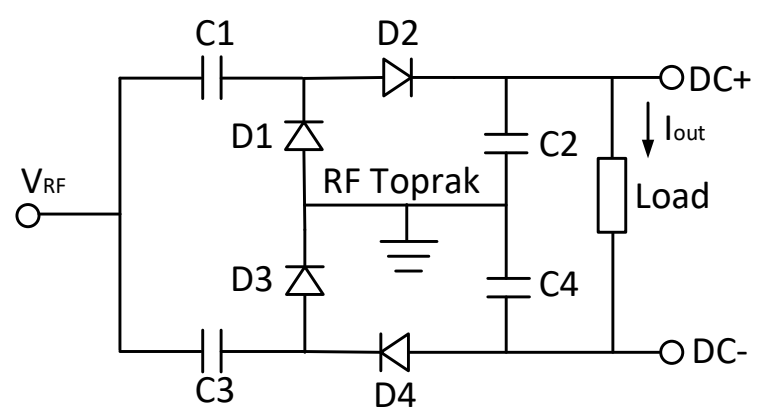

Şekil 2. Greinacher Tek Katlı Tam Dalga Doğrultucu

Şekil 2'de görüldüğü üzere D1, D2, D3 ve D4, zero-bias low-barrier Schottky diyotlarıdır, ortamdaki RF gücü düşük seviyelerde olmasına rağmen daha yüksek çıkış gerilimi anlamına gelir. Bu diyotlar ayrıca doygunluk derecesi yüksek bir akıma sahiptir, bu nedenle ek öngerilmeye gerek duymazlar. Birkaç mikroamperlik bias akımın ile çalışmaktadırlar. Şekil 2'de önerilen doğrultucu konfigürasyonunun tek dezavantajı ortaya çıkan daha yüksek seri dirençtir. Birincisi, eşleşen devrenin çıkışındaki indüklenmiş voltaj, DC bloklama kapasitörlerinden (C1 ve C3) geçer. Doğrulanan akım çıkışı daha sonra depolama kapasitörlerine pompalanır (C2 ve C4). Bu kapasitörlerde depolanan enerji doğrultucu düzgün durumuna ulaştığında, DC gücü yüke besler. Diğer doğrultucu devrelerle 
(Dickson veya Villard gibi) kıyasla, önerilen Greinacher doğrultucu simetriktir. Bu nedenle, her doğrultucu diyot, aynı giriş gücü ile harekete geçirilir. Buna ek olarak, bu simetrik yapı diyotlar tarafından üretilen harmonik içeriğin azaltılmasına yardımcı olur (hatta harmonikler birbirini iptal eder) (Olgun,Chen, 2012)[11].

$\mathrm{RF}$ hasat devresinin empedans uydurma aşaması, antenin doğrultucu devresine maksimum güç aktarımında kritik önem taşır. Bununla birlikte, uyumlandırma tasarımı zor olmaktadır çünkü doğrultucu diyotlar frekans, giriş gücü seviyesi ve yük direnci ile değișen karmaşık empedanslara sahip doğrusal olmayan elemanlardır. Bu nedenle sistem gereksinimleri belirlenerek en uygun uyumlandırma performansı için, bahsettiğimiz parametreler tasarımından önce belirlenmelidir.

$\mathrm{Bu}$ çalışmadaki RF enerji hasatlayıcı çalışma frekanları $2.4 \mathrm{GHz}$ (ISM) ve $2.7 \mathrm{GHz}$ (LTE) frekanslarını kapsamaktadır. Ayrıca, tasarım için yük direnci, $1 \mathrm{kohm}-10 \mathrm{kohm}$ ve $100 \mathrm{kohm}$ değişken yükleri için $10 \mathrm{dBm}-\quad-30 \mathrm{dBm}$ giriş güçünde çıkış voltajları incelenmiştir. Şekil 3'te uyumlandırılmış devrenin şematiği ve baskı devre gösterimi verilmiștir.

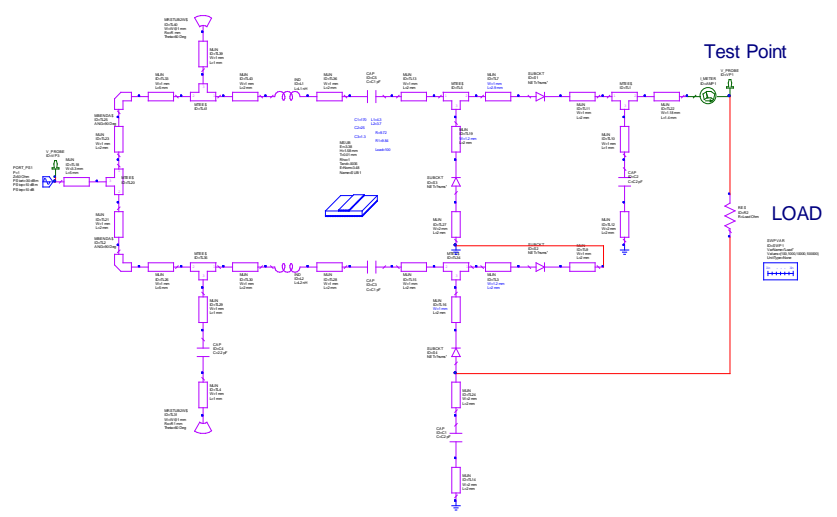

(a)

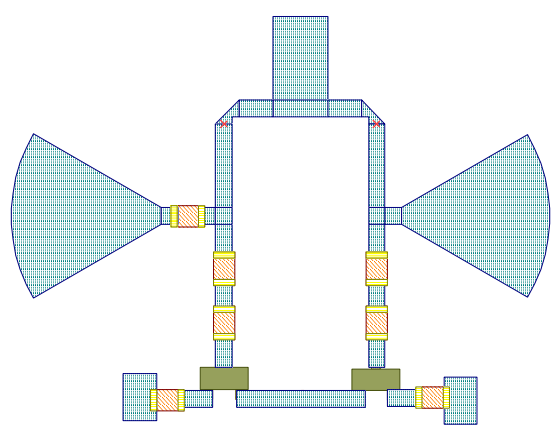

(b)

Şekil 3. Greinacher Tek Katlı Tam Dalga Doğrultucusu (a) Şematik ve (b) Baskı Devre Gösterimi
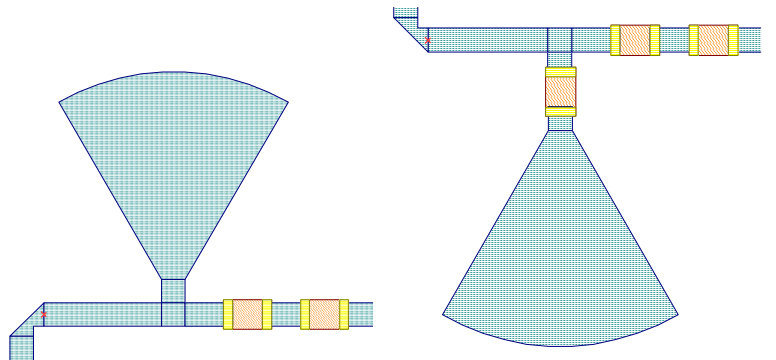

Şekil 4.: 2.4GHz Uyumlandırma Katı, $2.7 \mathrm{GHz}$ Uyumlandırma Katı

Şekil 4'teki devre yapısında uyumlama devresi tasarlanmış, bu uyumlama devresi L-C ve mikroşerit stub elemanlarını kapsamaktadır. Şekil 5'te devreye ait geridönüş (S11) kaybı gösterilmiștir. Kullanılan frekans bandı aralığında -10dB den daha az olduğu ve bu seviyenin geriyansıma kaybı için kabul edilebilir seviyede olduğu sanucuna varılmıștır.

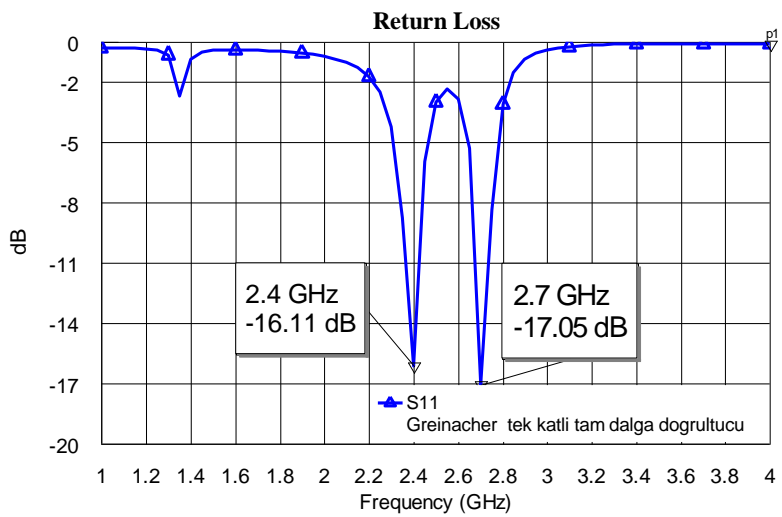

Şekil 5. Uyumlandırılmış tek katlı devrenin geri dönüş kaybi

Şekil 6'da çift bandlı doğrultucu devrenin girişine $30 \mathrm{dBm}$ ile $-30 \mathrm{dBm}$ arasında RF sinyal uygulanmıștır, $2.4 \mathrm{GHz}$ ile $2.7 \mathrm{GHz}$ arasında çıkış yükü ise $100 \mathrm{ohm}$ ile $100 \mathrm{Kohm}$ arasında değiştirilerek çıkış gücü ölçülmüştür.

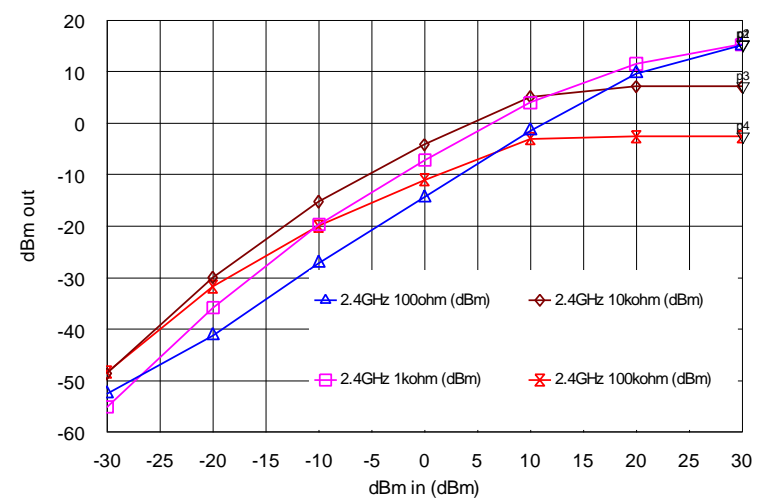

(a) 


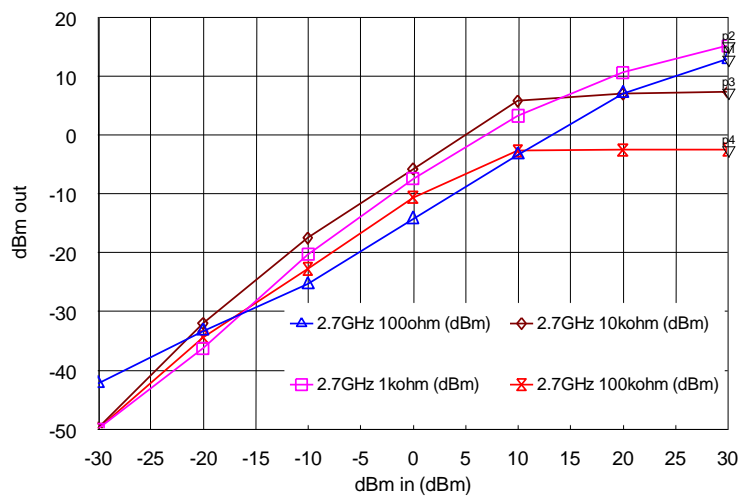

(b)

Şekil 6. $100 \mathrm{ohm}$ ile $10 \mathrm{Kohm}$ arasında Giriş gücüne göre çıkış gücü (a)2.4GHz, (b) $2.7 \mathrm{GHz}$

Tablo 1'de HSMS 2850 diyotlu Greinacher doğrultucu devre tasarımı üç farklı yük direnci için elde edilebilecek DC gerilim seviyesi verilmiștir. $-30 \mathrm{dBm}$ ile $+10 \mathrm{dBm}$ arasında RF giriş uygulanarak çıkış gerilimi elde edilmiştir.

Tablo 1. RF Giriș güç - DC Çıkış gerilim ( $\mathrm{P}_{\text {IN }}-\mathrm{V}_{\text {OUT }}$ ) Tablosu

\begin{tabular}{|c|c|c|c|}
\hline $\begin{array}{c}\text { Giriș RF Gücü } \\
\text { (dBm) }\end{array}$ & \multicolumn{3}{|c|}{ Vout (mV) (DC) } \\
\cline { 2 - 4 } & $\mathbf{1 K o h m}$ & $\mathbf{1 0 K o h m}$ & $\mathbf{1 0 0 K h m}$ \\
\hline-30 & 16 & 22 & 36 \\
\hline-20 & 69 & 124 & 214 \\
\hline-10 & 115 & 404 & 682 \\
\hline 0 & 182 & 1100 & 1750 \\
\hline 10 & 327 & 2959 & 4023 \\
\hline
\end{tabular}

Tablo 2'de tasarlanan doğrultucu devrenin performansı literatürdeki çalışmalarla kıyaslanmıştır. Elde edilen sonuçlara göre, greinacher doğrultucu devre tasarımı ile düşük empedans seçiminde veriminin daha yüksek, yüksek empedans seçiminde ise çıkış geriliminin arttığı gözlenmiştir. Bu konuda yapılacak iyileștirme çalışmalarında kullanılacak sistem yükünün belirlenerek devrenin verimi arttırlabilir.

Tablo 2. Doğrultucu Performans Karşılaștırması

\begin{tabular}{|c|c|c|c|}
\hline Kaynak & $\begin{array}{c}\text { Bu } \\
\text { Çalışmada }\end{array}$ & $\begin{array}{c}\text { (Papotto, } \\
\text { Carrara, } \\
\mathbf{2 0 1 1}\end{array}$ & $\begin{array}{c}\text { (Le, } \\
\text { Mayaram, } \\
\mathbf{2 0 0 8}\end{array}$ \\
\hline $\boldsymbol{f}(\mathbf{G H z})$ & 2.4 & 0.9 & 0.91 \\
\hline $\mathbf{P R F}_{\text {RF }}(\mathbf{d B m})$ & -10 & -22 & -15 \\
\hline $\mathbf{V}_{\text {rec }}(\mathbf{V})$ & 0.17 & 1 & 2.8 \\
\hline Kademe & 1 & 17 & 16 \\
\hline Teknoloji & HSMS & CMOS & CMOS \\
& 2850 & $90 \mathrm{um}$ & $150 \mathrm{um}$ \\
\hline
\end{tabular}

\section{Sonuçlar}

Yapılan çalışmada, yüksek frekanslı çift bant doğrultma devresi önerilmiştir ve güç spektrumu incelemesine dayalı ortamdaki $\mathrm{RF}$ enerjisiyi doğrultma amacıyla tasarlanmıştır. Elde edilen sonuçlar eşliğinde ortamda bulunan mevcut ISM ve LTE yada belirli kaynaktan yayllan elektromanyetik dalgaların doğrultularak verimli bir șekilde DC gerilim elde edilecektir. $\mathrm{Bu}$ amaçla çift katlı Greinacher doğrultucu devre tasarımı yapılmıştır. Devre girişinde yapılan L-C ve mikroşerit stub ile yapılan uyumlandırma ile devrenin $2.4 \mathrm{GHz}$ ve $2.7 \mathrm{GHz}$ frekanslarında uyumlu çalışabilmesi sağlanmıştır. Yapılan simülasyon sonuçlarına göre, $10 \mathrm{dBm}$ giriş gücüne karşıllk çıkışta $100 \mathrm{Kohm}$ 'luk yük direncinde $4,02 V^{\prime}$ luk bir DC gerilim elde edilmiştir. 0dBm'lik giriş gücünde ise LTE frekansında $100 \mathrm{ohm}$ ile $100 \mathrm{kohm}$ arasında değişen yük dirençlerinde -5 ile $-15 \mathrm{dBm}$ arasında güç değeri elde edilmiştir. Doğrultucudan elde edilen DC gerilim ile düşük güç tüketimine sahip sensörler ve sensör ağları beslenebilir ya da depolanarak pil ömrü uzatılabilir.

\section{Conflict of Interest / Çıkar Çatış̧ması}

Yazarlar tarafından herhangi bir çıkar çatışması beyan edilmemiștir.

No conflict of interest was declared by the authors.

\section{Kaynaklar}

Cheng, Y.-C., Chawathe, Y., LaMarca, A., Krumm, J., 2005. Accuracy characterization for metropolitan-scale Wi-Fi localization. Proc. Third Int. Conf. on Mobile Systems, pp. 233-245.

Curty, J.-P., Joehl, N., Krummenacher, F., Dehollain, C., Declercq, M., 2000. A model for m-power rectifier analysis and design. IEEE Trans. Circuits Syst. I, 52, (12), pp. 2771-2779.

Gilbert, J.M., Balouchi, F., 2008. Comparison of energy harvesting systems for wireless sensor networks. Int. J. Autom. Comput., 5, (4), pp. 334-347.

Hagerty, J.A., Helmbrecht, F.B., McCalpin, W.H., Zane, R., Popovic, Z.B., 2004. Recycling ambient microwave energy with broadband rectenna arrays. IEEE Trans. Microw. Theory Tech., 52(3), 1014-1024.

Le, T., Mayaram, K., Fiez, T., 2008. Efficient far-field radio frequency energy harvesting for passively powered sensor networks. IEEE J. Solid-State Circuits, vol. 43, no. 5, pp. 1287-1302.

Olgun, U., Chen, C.-C., Volakis, J.L., 2012. Design of an efficient ambient WiFi energy harvesting system. IET Microw. Antennas Propag., 6(11), 1200-1206.

Ostaffe, H., 2010. Power out of thin air: ambient RF energy harvesting for wireless sensors. http://powercastco.com/PDF/Power-Out-of-ThinAir.pdf

Papotto, G., Carrara, F., Palmisano, G., 2011. A 90-nm CMOS Threshold-Compensated RF Energy Harvester, SolidState Circuits. IEEE Journal of, 46(9), 1985 - 1997.

Paradiso, J.A., Starner, T., 2005. Energy scavenging for mobile and wireless electronics. IEEE Pervasive Comput., 4, (1), 18-27.

Rebello, J., 2010. Global wireless subscriptions reach 5 billion. Available: http://www.isuppli.com/Mobile-andWireless-communications/News/Pages/GlobalWireless-Subscriptions-Reach-5-Billion.aspx 
Sample, A., Smith, J, 2009. Experimental results with two wireless power transfer systems. Proc. IEEE RWS, 1618.

Yan, H., Montero, J. G. M., Akhnoukh, A., de Vreede, L. C. N., Burghart, J. N., 2005. An integration scheme for RF power harvesting. 8th Annu. Workshop Semiconductor Advances Future Electron. Sensors, Veldhoven, the Netherlands. 